
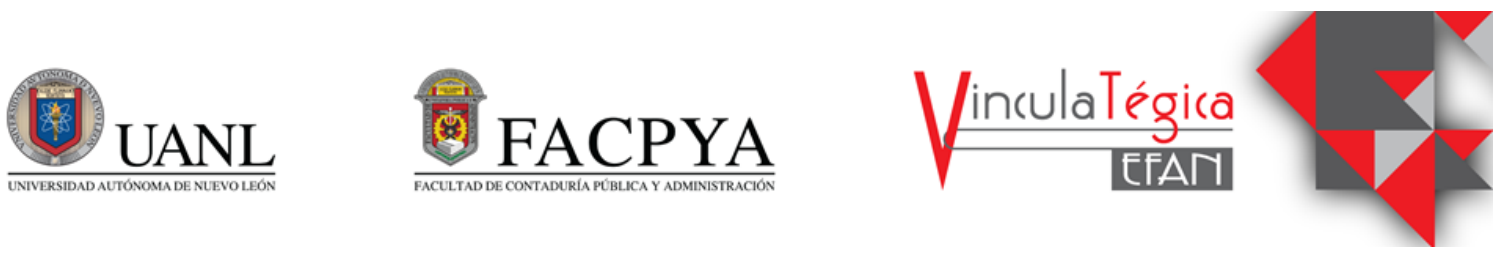

\title{
Estudio de las ventajas de las MiPyMES al adherirse a las redes sociales en Ciudad Victoria (Tamaulipas)
}

\author{
Juan José Rinconada Trejo ${ }^{1}$, José Rafael Baca Pumarejo ${ }^{2}$ y Julio César Macías Villarreal ${ }^{3}$ \\ ${ }^{1}$ Universidad Autónoma de Tamaulipas, rinconada.juanjose@gmail.com, Centro Universitario, Cd. Victoria, \\ Tamaulipas, (834)3181800. \\ ${ }^{2}$ Universidad Autónoma de Tamaulipas, rbaca@docentes.uat.edu.mx, Centro Universitario, Cd. Victoria, \\ Tamaulipas, (834)3181800. \\ ${ }^{3}$ Universidad Autónoma de Tamaulipas, jcmacias@docentes.uat.edu.mx, Centro Universitario, Cd. Victoria, \\ Tamaulipas, (834)3181800.
}

Información del artículo revisado por pares

Fecha de aceptación: junio-2021

Fecha de publicación en línea: diciembre-2021

DOI: https://doi.org/10.29105/vtga7.2-31

\begin{abstract}
Resumen
Abstract

La investigación tuvo como objetivo establecer las ventajas que ganan las mipymes al adherirse a las redes sociales. Además, se establecieron dos objetivos específicos: determinar la intensidad de uso que dan las mipymes victorenses a las redes sociales e identificar cuáles fueron las dimensiones de mayor y menor impacto en las razones para adherirse a las redes sociales de las empresas analizadas. Para ello se utilizó el tipo de investigación cuantitativa, mientras que el diseño fue no experimental. La población estuvo formada por 85 mipymes de Ciudad Victoria, Tamaulipas. Como resultado de este estudio se evidenció que las dimensiones evaluadas, guardan una relación lineal con la variable dependiente Ventajas de Adherirse a las Redes Sociales (adherSN) y tienen una influencia positiva en la misma, porque se evidenció que todas contribuyen a incrementar su valor debido a las ventajas que representa incorporar sus negocios al uso y aplicación de las redes sociales. Las ventajas al usar las redes sociales son: identificar oportunidades para crear o mejorar productos o servicios, obtener nuevos clientes, compartir la información con los

The objective of the research was to establish the advantages that MSMEs gain by joining social networks - setting two specific goals: to determine the intensity of use that Victorenses MSMEs give to social networks. And identify the dimensions with the most significant and most minor impact on the reasons for joining the social networks of the companies analyzed. The study type was quantitative research, while the design was non-experimental. The population consisted of 85 MSMEs from Ciudad Victoria, Tamaulipas. The study evidenced a linear relationship between the dependent variable: Advantages of Adhering to Social Networks (adherSN), and the set of independent variables, positively influencing it. The advantages of using social networks are: identifying opportunities to create or improve products or services, obtain new customers, share information with consumers and get feedback from them, promote the image, be close and in real-time with customers through adequate communication and innovation, access to low-cost marketing, among others. The study yielded favorable results on the perception that MSMEs owners have about the advantages of joining social networks and strengthening their business processes.
\end{abstract} consumidores y obtener retroalimentación de ellos, promover la imagen, estar cercano y en tiempo real con los clientes mediante una adecuada comunicación e innovación, acceder a un marketing

Keywords: MSMEs, Social Networks, Marketing.

JEL: M310, 0330. 
de bajo costo, entre otras. El estudio arrojó resultados favorables sobre la percepción que los dueños de mipymes tienen sobre las ventajas para adherirse a las redes sociales y fortalecer los procesos de sus negocios.

Palabras clave: Mipymes, Redes Sociales, Mercadotecnia.

\section{INTRODUCCIÓN}

El presente estudio da a conocer que las redes sociales son un nuevo canal de negocios para las empresas, en el que se puede captar a nuevos clientes además de muchos otros beneficios, tales como: canales de comunicación interna y externa, selección de recursos humanos, colocar y hacer reconocida su marca en el mercado, redes de negocios, entre otros.

Utilizar las redes sociales para realizar una campaña, puede ser la gran diferencia que eleve al producto o servicios que se promueve.

Esto es posible por el elevado número de usuarios de las redes sociales; y dentro de ese universo seguramente habrá quienes se interesen por la empresa.

Es increíble que aún en estos tiempos existan personas que no sepan de la gran oportunidad que representa el uso de estas herramientas. Esta investigación fomenta la iniciativa de utilizar las nuevas tendencias en redes sociales que ayudan a aumentar la competitividad y por ende ayudan al crecimiento económico de la región. Además, la mipyme reconocerá la importancia del manejo de las redes sociales ya que trae ciertas ventajas como acceder a otros mercados que su establecimiento físico se los impide.

El presente estudio es oportuno para la época actual ya que las Redes Sociales (RRSS) son una realidad que ha hecho que cambie la forma en que las empresas interactúan con sus clientes, logrando con ello que sea más directa y ágil la comunicación, al igual que ha hecho que la mercadotecnia y publicidad tengan estrategias atractivas y con resultados contundentes.

Además, el costo de utilizar las redes sociales para anunciarse es más bajo, que hacerlo por otros medios tradicionales, como periódicos, revistas y televisión, etc.

\section{MARCO TEÓRICO}

\subsection{Redes sociales}

Franco, Haase y Pereira (2016), mencionan que desde la perspectiva de las redes sociales y con el desarrollo de la Web 2.0, las empresas y a la población en general han obtenido nuevas oportunidades y beneficios, debido a la facilidad de la comunicación y la gran velocidad con la que se transmite la información. Una de las mayores oportunidades fue el advenimiento de nuevas aplicaciones de red en línea conocidos como redes sociales (Franco et al, 2016; Dokyun et al, 2018; Sánchez \& De la Garza, 2018). En la literatura, las diferentes definiciones se han utilizado para describir el significado de las redes sociales, tales como los lazos sociales, interpersonales, las relaciones personales (Borgerson y Miller, 2016; Dokyun et al., 2018) mencionan que la red social es una relación de las personas que están conectadas a través de interacciones sociales basadas esencialmente en los intercambios sociales, sino que también puede contener el intercambio de información y de negocios.

Mientras que Yang et al. (2019), definen a las RRSS como un grupo de conexiones específicas entre un grupo de personas definidas, y, además, las características de esas conexiones en su conjunto pueden ser utilizados para interpretar el comportamiento social de las personas involucradas dentro de la red social. Las redes sociales contienen información acerca de los perfiles de los individuos y su red, que puede ser utilizado para diversos negocios.

Los beneficios de la participación en las redes sociales sólo existen si las empresas son capaces de entender el poder de la conducta 
colectiva para estimular un cambio positivo en los negocios (Bradley, 2017). Las empresas pueden llegar a innumerables clientes potenciales, clientes a los que no pueden acceder de otra manera, mientras que el ahorro en campañas publicitarias es enorme (Al-Badi \& Al-Qayoudhi, 2014).

La red social es atractiva para las empresas por su facilidad de uso, y porque permiten la comunicación en tiempo real (Bradley, 2017). Borgerson y Miller (2016), mencionan que las redes sociales permiten a dueños de mipymes evaluar los patrones de comportamiento de sus usuarios, ver qué contenidos complacen a sus actitudes y comportamientos y que comunidad provoca más publicidad. Las empresas pueden discutir asuntos y preocupaciones de manera virtual y tomar las mejores decisiones de negocio. Otra ventaja aparece cuando una empresa quiere introducir nuevos productos, que en una primera fase se pueden presentar a través de la demostración virtual y pedir la opinión de las personas a través de las redes sociales. La capacidad de innovación de las mipymes es esencial para obtener una ventaja competitiva en un mercado en constante actualización, donde el desenvolvimiento continuo de los procesos de nuevos productos es la clave para el crecimiento y la rentabilidad (Revilla, Perez-Luno \& Nieto, 2016).

Puesto que, debido a sus limitados recursos, las mipymes utilizan una variedad de fuentes y están entrelazadas a redes diferentes para obtener la información que requieren para desarrollar su estrategia y organizar de mejor manera su entorno por lo que las redes sociales y las mipymes se mantienen al día con los cambios constantes en la economía, ya que se aprovechan de las oportunidades para innovar los productos y servicios (Ayyagari et al., 2017).

Debido que las limitaciones para el uso de la tecnología de red social son pocas, las mipymes pueden trabajar de la misma manera que las empresas multinacionales, sin la necesidad de contar con grandes recursos (Franco et al., 2016). De igual manera el internet puede hacer que sea más fácil para una empresa expandir su alcance y ampliar su actividad a través de la penetración de mercado o el desarrollo de productos. De acuerdo con Bradley (2017), las relaciones formadas a través de Internet pueden estimular el incremento en las ventas y crear oportunidades para proporcionar innovadores productos y servicios. Adicionalmente, es importante que las empresas tengan una capacidad de innovación que las lleven a incrementar su competitividad e incluso, que sus mejoras se extiendan a todo el comercio y la economía en general, a través de la diseminación del conocimiento a otras mipymes.

\subsubsection{Redes sociales en Estados Unidos}

De acuerdo con un análisis realizado por las empresas Price Waterhouse Coopers (PwC) e Interactive Advertising Bureau (IAB), con respecto a la inversión en publicidad en internet que realizan las empresas en Estados Unidos, en 2014, por primera vez en este país, la publicidad por internet desbancó a la publicidad televisiva. Este informe da a conocer que la publicidad en línea tuvo un crecimiento del $17 \%$, superior a la que la publicidad televisiva obtuvo ese año (Goldberg, 2014).

La empresa IAB indicó que el incremento en la publicidad en internet se debió en gran parte al crecimiento de usuarios de dispositivos móviles, así como a la motivación de las empresas por adquirir publicidad en esta vía (Goldberg, 2014).

\subsubsection{Redes sociales en México}

En cuanto a la publicidad en internet, la empresa Interactive Advertising Bureau, realizó una comparación entre Estados Unidos y México y mencionó que, si bien hay un avance para consolidar la publicidad en línea, hay aún áreas de oportunidad que deben aprovechar las empresas (Medina, 2014).

Las actuaciones que hacen las empresas mexicanas que usan Internet principalmente lo hacen con la intención de que sus ventas se vean incrementadas, para llegar a nuevos clientes potenciales, para darse a conocer en cualquier parte del mundo, para crear fidelidad y credibilidad del cliente hacia la empresa y para dar a conocer sus nuevos productos o servicios (Santana, 2015); Alvino (2021) 
menciona los resultados del Digital 2021 Global Overview Report, publicado por We are Social y Hootsuite: "Actualmente en México hay 100 millones de personas que hacen uso de las diversas plataformas de redes sociales, lo que representa un $77.2 \%$ de la población mexicana activa en internet. Para enero del 2021, el uso de las redes sociales en México se incrementó $12,4 \%$, es decir 11 millones de usuarios nuevos. Del total de usuarios que utilizan las redes sociales el 98.9\% acceden a través de sus dispositivos móviles".

Martínez (2018) publicó un artículo en 2018 el Diario El Economista en el que cual menciona que, durante el sexenio de Enrique Peña Nieto, se realizó una reforma constitucional en materia de telecomunicaciones, a la cual se adicionó como un derecho para los mexicanos, el acceso a los servicios de internet. Al concluir la administración peñista, la tasa de crecimiento promedio anual de usuarios de internet fue de $11.5 \%$, con más de 79 millones de usuarios en 2017, a diferencia de los 51 millones de usuarios reportados en 2013.

El tiempo de conexión del internauta fue de 8 horas y 12 minutos al día. El registro de los horarios de mayor tráfico de internet para usuarios de México es de las 6:00 a 9:00 horas y entre las 21:00 y 24:00 horas. Siendo el horario con menor cantidad de usuarios, entre las 12:00 y 14:00 horas.

La principal actividad que realizan los mexicanos es acceder a redes sociales. El 89\% de los internautas utiliza el internet para navegar en sus redes sociales. Además, mencionó que los mexicanos que cuentan con acceso a internet poseen en promedio 5 redes sociales. Casi la totalidad de los internautas del país cuentan con Facebook, y 9 de cada 10 tienen WhatsApp. Facebook es la red social dominante en México ya que el $98 \%$ de la población mexicana utiliza esta red, WhatsApp es la segunda más utilizada con una tasa de usuarios del 91\%; quedando en tercer lugar YouTube con 82\%; posicionada en cuarto lugar queda la red social Twitter con un $57 \%$ quedando así en quinto lugar de las redes más utilizadas en México Instagram con un 47\%. Cifras calculadas por Estadística Digital
INFOTEC y Asociación de Internet con base en información de CONAPO e INEGI.

2.2 Pequeñas y medianas empresas (Pymes) En algunos países de América Latina, Europa y Norteamérica, se piensa que las siglas SME, MSMES, MIPYME, MYPE, PYMI O MIPYMEA definen las empresas micro, pequeña y medianas empresas, con ventas anuales totales, la utilidad anual, el patrimonio, los activos y el capital, en cantidades que se definen según los contextos de cada país. Ello también puede depender del capital humano, las ventas anuales totales, la utilidad anual, el patrimonio, los activos y el capital suscrito (Saavedra, Camarena \& Tapia, 2017).

El Banco Mundial (2016) afirma que las microempresas en América Latina son una fuente fundamental de sostenimiento para la sociedad $y$ son grandes productoras de empleos para las familias. En consecuencia, impactan en el producto interno bruto (PIB) y por ende su economía y bienestar. Por lo que las investigaciones acerca estos negocios (small enterprises) deben analizar su aporte conjunto al bienestar laboral y al desarrollo socioeconómico de las familias en el contexto regional (Arceo-Moheno et al., 2019).

$\mathrm{Si}$ una microempresa del sector informal representa una solución económica que beneficia a su dueño y a su familia, no debe evaluarse su desempeño y dinamismo de la misma forma como se hace en el caso de una gran empresa, porque perdería su visibilidad y su pertinencia local y regional (Marc, 2015). Para apreciar la importancia económica y social de la microempresa en la generación de empleo, servicios e ingresos de las regiones y localidades, debe analizarse en su totalidad macroeconómica, no en su individualidad o particularidad microeconómica (ArceoMoheno et al., 2019). Para muestra basta un botón: el $97 \%$ de los municipios de Colombia dependen política, social y económicamente de la microempresa (Muñoz \& Mayor, 2016).

De acuerdo con Arrubla (2016), es necesario hacer una separación entre la gran empresa y la mipyme, ya que, muchas de las 
microempresas familiares no separan la contabilidad de la empresa de las cuentas del hogar, incluso los microempresarios manejan sus unidades de negocio con criterios más emocionales que propiamente dichos de una racionalidad económica-empresarial. Otra de las dificultades de acuerdo con Ayyagari, Demirguc-Kunt \& Maksimovic (2017), Bocconcelli et al. (2018) y Torm (2020), es que "en América Latina para medir con la misma racionalidad la mediana y la gran empresa respecto a la microempresa es que las primeras no tienen la misma libre movilidad en el mercado para entrar y salir, en diferencia con la segunda, por tamaño de planta, responsabilidad financiera y comercial o por falta de registro". Según Torm (2020), una práctica entre microempresas es reducir los costos de producción y de servicios, ya que no asumen en su totalidad los costos salariales y de la seguridad social.

La CEPAL (2020) afirma que "en América Latina el progreso de la microempresa en todas las economías depende, especialmente, de las redes empresariales y del clúster", así como también de los subsidios que ofrece el gobierno, de los impulsos comerciales, siendo importante también las políticas públicas que se enfocan a fomentar las empresas, de los tejidos empresariales públicos y privados que puedan llegarse a formar entre las empresas (Bocconcelli et al., 2018).

\subsubsection{Composición de la mipyme en América Latina}

Este tipo de empresas no alcanzan niveles de competitividad significativos en América Latina debido a que no existe una vinculación del sector industrial con la investigación en desarrollo tecnológico, y tampoco del sector empresarial con la Academia, de forma que no se da el círculo virtuoso de mutuo fortalecimiento para incrementar el valor agregado de la producción y los nuevos productos. Por lo que se puede decir que la investigación y desarrollo empresarial es muy escasa en América Latina, lo que implica una debilidad competitiva de la empresa debido a inferiores niveles de calidad en la educación
(Granda \& Hamann, 2015). En este escenario Muñoz y Mayor (2016), mencionan que en la región referida las universidades tienen una escasa producción de patentes comparadas con universidades de otros continentes, según el índice de Shangai Academic Ranking Universities.

En 2014, las primeras universidades latinoamericanas en destacar en el ranking de las 500 mejores a nivel global eran: Universidad de Sao Paulo, Brasil (101-150), Universidad de Buenos Aires, Argentina (151200), Universidad Autónoma de México (201300), y la Universidad de Chile (401-500). Estas circunstancias afectan a un contexto donde $90 \%$ o más del total de la mipyme latinoamericana es microempresa (Chiatchoua $\&$ Porcayo, 2017). Y lo más importante es que es irrefutablemente estas empresas en América Latina son grandes generadoras de empleo para mano de obra poco calificada; de allí que las mipymes representan entre el $80 \%$ y el 90\% del empleo total (Chiatchoua \& Porcayo, 2017).

\subsubsection{Clasificación de la mipyme en Estados Unidos}

En Estados Unidos, las "Small and Medium Enterprises" (SME) se caracterizan porque son medianas empresas. También se encuentran las microempresas familiares cuya proyección de crecimiento es considera baja; no obstante, también hay empresas que tienen una proyección alta y para ello, buscan esquemas para su crecimiento, incluyendo programas de apoyo del gobierno.

Otra ventaja de estas empresas medianas, es que, debido a su tamaño, se manejan diferente a un empresa grande, ya que sus procesos, tanto administrativos como operativos y sus utilidades son más fáciles de manejar, lo que puede redundar en un desarrollo mayor (Chiatchoua \& Porcayo, 2017). Otra de las principales características de las mipymes estadounidenses para nuevas inversiones y necesidades de crecimiento es el mercado bursátil. La Bolsa de Valores de Nueva York ofrece, por ejemplo, índices bursátiles especializados en las SME, tales como el Nasdaq y el Dow Jones, para más de 7000 empresas de pequeña y mediana 
capitalización (Muñoz \& Mayor, 2016).

\subsubsection{Mipymes en México}

La evolución de las mipymes en México comenzó entre los años 30 y los 40 cuando se dio importancia a los sectores del acero, vidrio, alimentos y bebidas, cemento, textil, entre otros, gracias a la industrialización impulsada en ese entonces por los presidentes Manuel Ávila Camacho y Miguel Alemán Valdés, surgiendo las mipymes en estos sectores (Chiatchoua \& Porcayo, 2017). Las mipymes mexicanas nacieron para el año de 1950, que se desarrollaron en el escenario proteccionista del gobierno, que tenían como característica principal a empresarios con poca preparación, pero de mucha tenacidad y dedicación (Chiatchoua \& Porcayo, 2017). Al final del siglo XX, nacieron nuevos métodos, innovaciones $\mathrm{y}$ estrategias en la administración empresarial, en los años 80 a estas empresas les fue muy complicado adaptarse al cambio; hubo un mayor desarrollo en el comercio, llevando esto a que un sector de los empresarios hiciera a un lado la producción de bienes y servicios, para enfocar su fuerza de trabajo en la importación, considerando con ello que es más rentable y el riesgo es mucho menor (Chiatchoua \& Porcayo, 2017).

A las mipymes se les reconoce como la pieza clave en la economía y el desarrollo social de nuestro país, toda vez que son la fuerza empresarial más fuerte, así como generadoras de empleos (Santana, 2015).

De acuerdo con datos oficiales del Instituto Nacional de Estadística y Geografía (INEGI), en México existen 4.2 millones de unidades económicas. De ese universo, el 99.8\% son consideradas Micro, Pequeñas y Medianas Empresas (Mipymes), las cuales aportan $42 \%$ del Producto Interno Bruto (PIB) y generan el $78 \%$ del empleo en el país (Forbes, 2018).

\subsection{Razones para adherirse a las redes sociales.}

En el ámbito internacional existe el reto constante por la permanencia, creación y desarrollo de las Mipymes como consecuencia de la alta aportación en los indicadores económicos. Otro reto se tiene que ver con el aumento en el grado de desaparición de este tipo de negocios, ya que la mayoría de estas empresas no poseen una contabilidad objetiva que permita evaluar el rendimiento y estar en posibilidades de advertir su expansión o desaparición CEPAL (2020).

Investigadores de este campo consideran el crecimiento como la medida de rendimiento de mayor relevancia en las pequeñas empresas (Torm, 2020) y el crecimiento del empleo es otro aspecto por considerar cuando se intenta medir el rendimiento empresarial CEPAL (2020).

Se han creado variadas metodologías y herramientas para medir el rendimiento de las empresas, existen de tipo financiero, operativo y de eficacia; algunas de ellas son objetivas, es decir, se extraen de cifras o datos producto por ejemplo de sistemas contables y presupuestales o del sistema de mando integral (Ballina Ríos et al., 2016); otras son más bien subjetivas, ya que miden el rendimiento a través de la percepción interna y externa de la empresa. En general, puede decirse que hasta el momento no existe acuerdo respecto de un indicador generalizado para medir el rendimiento (Pelekais \& Ortega, 2020). Para esta investigación se ha tomado como referencia el trabajo de Franco, M., Haase, H., \& Pereira, A. (2016). Cuyo título en inglés fue "Empirical study about the role of social networks in SME performance". Y se evalúa solo la percepción de las mipymes al usar redes sociales y medir las razones para adherirse a las redes sociales.

Las razones para adherirse a las redes sociales a su vez se evaluaron mediante las siguientes dimensiones:

2.3.1 IOP. Variable de la identificación de las oportunidades (Identificaction of Opportunities) que resaltan las razones asociadas a unirse a las redes sociales con el fin de identificar y establecer nuevos puntos de comunicación fuera de la empresa.

2.3.2 SI. Compartición de la información (Sharing Information). Este factor se compone de cuatro variables, como adquirir nuevos clientes, seguimiento a los clientes existentes, 
desarrollando relaciones, identificación de nuevas oportunidades de negocio, atención a la competencia vía actualización de información.

2.3.3 CI. Comunicación e Innovación (Communication and Innovation). $\mathrm{Se}$ relaciona con aumento de la credibilidad y el prestigio de la marca, promoción de la innovación, productos personalizados, soporte las 24 horas, comunicación en tiempo real, todo esto para que estar cerca del cliente o cliente potencial, siempre satisfaciendo los gustos y deseos de cada caso específico.

2.3.4 RDC. Reducción de Costos (Cost Reduction). Relacionados a la posibilidad de reducir los costos de marketing y publicidad de la empresa. Las Redes sociales son una herramienta de marketing de bajo costo, dando a conocer a la empresa sin fronteras.

2.3.5 MKT. Mercadotecnia (Marketing). Entender las opiniones de los clientes y fidelizarlos facilita la internacionalización de la empresa, pudiendo aprender qué grupos de clientes coinciden con la empresa y, por lo tanto, buscar la expansión del mercado.

2.3.6 adherSN. Ventajas de Adherirse a las Redes Sociales. Variable que evalúa las percepciones de los empresarios acerca de las ventajas que representa adherirse a las redes sociales para potenciar la comunicación y la promoción de sus negocios.

\section{MÉTODO}

De acuerdo con la presente investigación, podemos definir que es una investigación de tipo descriptiva, y se utiliza el método cuantitativo ya que se procedió a recolectar información, el diseño es no experimental ya que se basó en la aplicación de 85 cuestionarios a mipymes comerciales y de servicios de Ciudad Victoria, Tamaulipas. Así mismo es una investigación documental, debido a que se utilizaran revistas, internet y bases de datos, además en artículos de revistas y libros acerca de las redes sociales y las mipymes, así como las teorías aplicadas y las distintas aportaciones de diversos autores que brindan información para esta investigación.

Para lograr los objetivos propuestos

para esta investigación, se tomó el cuestionario que fue elaborado por Franco et al. (2016) a partir de otros estudios científicos y evalúa las razones para adherirse a las redes sociales. El cual estaba conformado por 20 preguntas que cuentan con escalas tipo Likert de cinco puntos, que van desde "1 Completamente en desacuerdo" a "5 Completamente de acuerdo". Por lo tanto, se cumple fielmente con la norma de Validez de Contenido del Instrumento, en donde se establece la recomendación sobre el análisis del instrumento de recolección por tres o cinco expertos aproximadamente.

Se realizó una prueba de confiabilidad Alpha de Cronbach, y a partir de los datos obtenidos se obtuvo la confiabilidad y cuya fórmula matemática es la siguiente: $\alpha=\frac{K}{K-1}\left[1-\frac{\sum S_{i}^{2}}{S_{T}^{2}}\right]$

Donde:

$\mathrm{K}$ : El número de ítems

$\sum \mathrm{Si} 2$ : Sumatoria de Varianzas de los Ítems

ST 2: Varianza de la suma de los Ítems $\alpha$ : Coeficiente de Alfa de Cronbach

Tabla 1. Fiabilidad Alpha de Cronbach

\begin{tabular}{|c|c|}
\hline & Alpha de Cronchbach obtenido \\
\hline $\mathbf{Q}$ & 0.804318439 \\
\hline
\end{tabular}

Fuente: Elaboración propia

Tabla 2. Rangos de magnitud

\begin{tabular}{l|l|l}
\hline Muy alta & 0.81 & 1.00 \\
Alta & 0.61 & 0.80 \\
Moderada & 0.41 & 0.60 \\
Baja & 0.21 & 0.40 \\
Muy baja & 0.01 & 0.20 \\
\hline Fuente: (Kerlinger \& Lee, 2002).
\end{tabular}

Después de realizar el análisis, de acuerdo con la tabla 2, se obtuvo un índice de confiabilidad alto.

\subsection{Cálculo del tamaño de la muestra}

Para esta investigación se utilizó la base de 
datos $\frac{\frac{z^{2} \times p(1-p)}{e^{2}}}{1+\left(\frac{z^{2} \times p(1-p)}{e^{2} N}\right)}$

Directorio Estadístico Nacional de Unidades Económicas (DENUE) del Instituto Nacional de Estadística y Geografía (INEGI). Al aplicar lo filtros de sector comercial (al por menor) y otros servicios (excepto actividades gubernamentales) en dicha base de datos, se obtuvo que son 6,857 mipymes la población de estudio en la ciudad. De las cuales las mipymes comerciales son las de mayor cantidad con 4,345, es decir, un $63.37 \%$ de la población de estudio. Mientras que las de otros servicios fueron 2,512, es decir, el $36.63 \%$ del total. La muestra del presente estudio se obtuvo a partir de la siguiente fórmula:

Tabla 3. Valores de la fórmula del cálculo de la muestra

\begin{tabular}{cll}
\hline $\mathrm{n}$ & Tamaño de la población & 6,857 \\
$\mathrm{e}$ & Margen de error & 8.9 \\
$\mathrm{p}$ & $\begin{array}{l}\text { Proporción de la población con } \\
\text { la característica deseada (éxito) }\end{array}$ & $90 \%$ \\
$\mathrm{z}$ & $\begin{array}{l}\text { Cantidad de desviaciones } \\
\text { estándar que una proporción }\end{array}$ \\
& determinada se aleja de la media \\
\hline Fuente: &
\end{tabular}

Con los parámetros de la tabla anterior se obtuvo la muestra de nuestra investigación, resultando 85 .

\subsection{Recolección de datos}

Con el fin de obtener los datos necesarios para la investigación, la información de este estudio fue procesada. A partir de dicha información, se elaboraron tablas de cálculo electrónicas mediante las cuales se crearon las bases de datos para el estudio de la influencia de las redes sociales en las empresas victorenses.

\section{RESULTADOS}

El cuestionario utilizado midió a través de 3 indicadores dicotómicos agregados a los indicadores de Franco et al. (2016) la intensidad de uso de las redes sociales con las opciones de baja, media y alta frecuencia de uso.

Gráfica 1. Intensidad de uso de las redes sociales

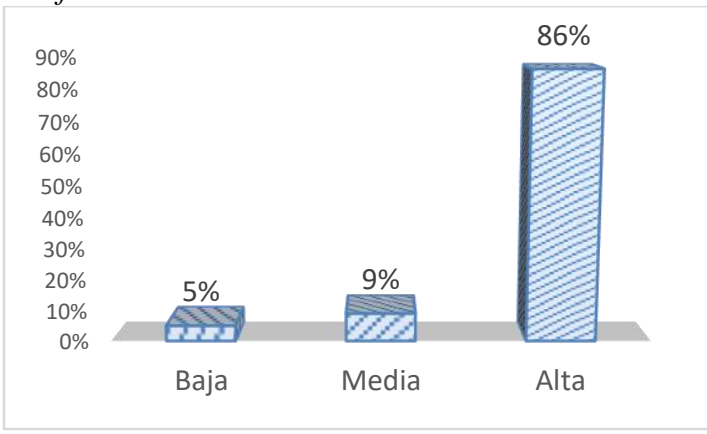

Fuente: Elaboración propia

Como se muestra en la Gráfica 1, se evidenció que, de acuerdo con la percepción de los encuestados, existe una alta intensidad de uso de las redes sociales, ya que el $86 \%$ de los ya mencionados optaron por responder a los indicadores con las opciones que representaban una mayor intensidad. Se hizo una regresión lineal (Tabla 4) que incorporó análisis multivariado para describir los niveles de impacto de las dimensiones ya descritas sobre la variable dependiente Ventajas de Adherirse a las Redes Sociales, y fundamentar los hallazgos.

Tabla 4. Resultados de la regresión 
- regress adherSN IOP SI CI RDC MKT

\begin{tabular}{r|rrc} 
Source & SS & df & MS \\
\hline $\begin{array}{r}\text { Model } \\
\text { Residual }\end{array}$ & 5.70273544 & 79 & .072186525 \\
\hline Total & 17.2235294 & 84 & .205042017
\end{tabular}

Number of obs $=\quad 85$

$F(5,79)=31.92$

Prob $>\mathrm{F}=0.0000$

R-squared $\quad=0.6689$

Adj $\mathrm{R}$-squared $=0.6479$

Root MSE $=.26868$

\begin{tabular}{r|rrrrrr}
\hline adherSN & Coef. & Std. Err. & $t$ & P > t I & [95\% Conf. Interval] \\
\hline IOP & .231914 & .0375066 & 6.18 & 0.000 & .157259 & .306569 \\
SI & .2660658 & .0946067 & 2.81 & 0.006 & .0777559 & .4543757 \\
CI & .2664051 & .0821565 & 3.24 & 0.002 & .1028767 & .4299336 \\
RDC & .1104858 & .0458255 & 2.41 & 0.018 & .0192724 & .2016992 \\
MKT & .1621835 & .0574818 & 2.82 & 0.006 & .0477689 & .2765982 \\
_Cons & .0031654 & .4639028 & 0.01 & 0.995 & -.92021 & .9265408 \\
\hline
\end{tabular}

Fuente: Elaboración propia 
Se utilizó el paquete estadístico Stata para procesar la base de datos y se planteó un modelo lineal donde las dimensiones IOP, SI, CI, RDC, MKT revelaron tener una influencia significativa sobre adherSN, la variable dependiente.

El modelo quedó explicado al $66.89 \%$, con $\operatorname{Prob}>\mathrm{F}=0.0000$ y 85 reactivos analizados, como se muestra al detalle en la Tabla 4.

Así mismo en Stata se hizo una prueba de colinealidad evidenciando en función de la inflación de la varianza (VIF), la ausencia de colinealidad entre las variables independientes como se muestra en la Tabla 5, dado que todas son inferiores a 10 .
Tabla 5. Inflación de Varianzas

. vif

\begin{tabular}{r|rr} 
Variable & VIF & 1 VIF \\
\hline CI & 1.52 & 0.655885 \\
SI & 1.28 & 0.782578 \\
MKT & 1.25 & 0.797001 \\
RDC & 1.20 & 0.833866 \\
IOP & 1.12 & 0.896371 \\
\hline Mean VIF & 1.27 &
\end{tabular}

Los resultados permitieron estudiar el modelo que analiza la relación lineal entre las ventajas de Adherirse a las Redes sociales a partir de las cinco dimensiones exploradas por el instrumento de recolección de datos Franco (2016) representadas por Oportunidad de la información (IOP), Compartición de la información (SI), Comunicación e Innovación (CI), Mercadotecnia (MKT) y Reducción de Costos (RDC).

Figura 1. Modelo Relacional de Variables

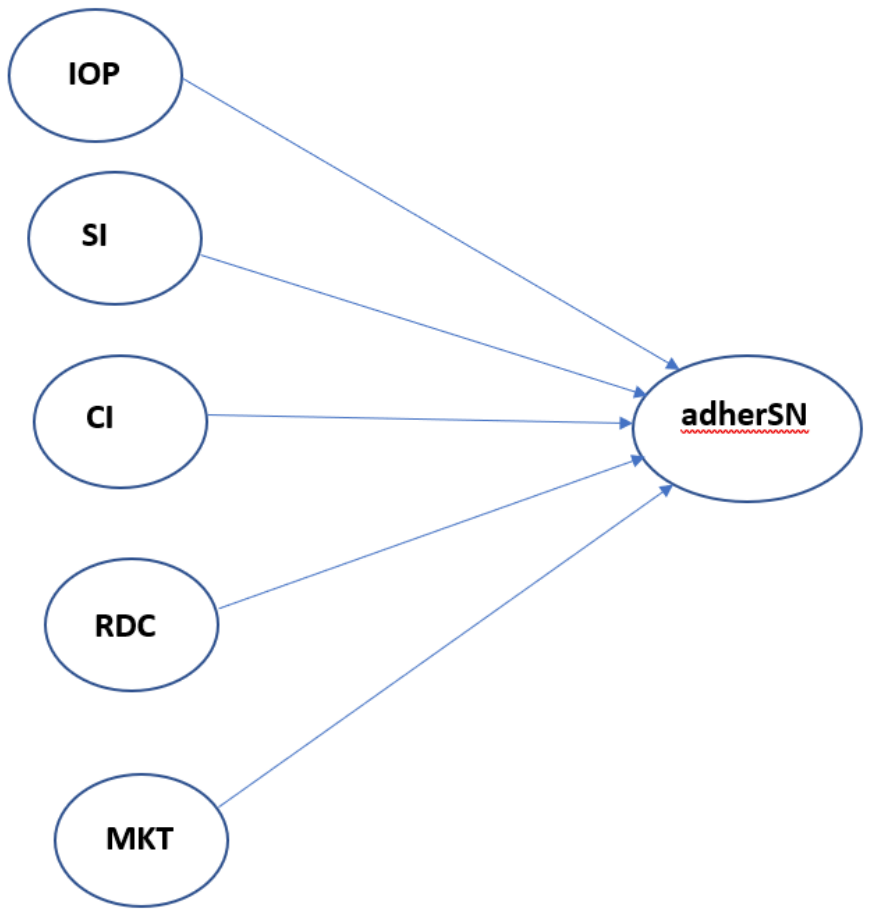

Fuente: Elaboración propia 
Este modelo de regresión muestra la percepción de los encuestados en relación con las cinco dimensiones que se evalúan; ubicó de manera contundente a la Comunicación e Innovación (CI) y a Compartición de Información (SI) como las dimensiones de mayor impacto en las ventajas de adherirse a las Redes Sociales, puesto que son las que más contribuyen a su aumento ya que, por cada .2664 de CI y por cada .2660 de SI, respectivamente, aumenta una unidad la ventaja de adherirse a las redes sociales. Seguido de ellas, en tercer lugar, se evidenció la dimensión de Oportunidad de la Información (IOP) la cual contribuye al modelo con .2319 de su valor por cada unidad adicional en la variable dependiente. En general, el modelo evidencia las ventajas de adherirse a las redes sociales como permitir la atracción de nuevos clientes, tener un mayor contacto con los mismos, identificar nuevas oportunidades de negocios y estar al tanto del mercado y los competidores. Por lo que la información obtenida por medio de las redes sociales es de gran ayuda para el buen funcionamiento de las mipymes.

En contraste, la dimensión que se percibió con el menor impacto según el modelo lineal fue la Reducción de Costos (RDC) como lo evidencia la Tabla 4, dado que contribuye con el mínimo valor de impacto sobre la variable dependiente analizada con solo .1104 por cada punto que aumenta la variable dependiente, esta dimensión evaluó la oportunidad de obtener un marketing de bajo costo, así como también la reducción en los costos de comunicación, cuestión que los gerentes no aquilataron lo suficiente como razón sólida para adherirse a las redes sociales. Además, las variables independientes evaluaron mediante sus indicadores la credibilidad y prestigio de la empresa, presentar nuevas ideas y/o innovaciones en sus productos o servicios, presentar los productos o servicios ofrecidos a un mayor número de clientes potenciales, comunicación en tiempo real y además que les ayuda en el desarrollo de nuevos productos personalizados o servicios de calidad. Finalmente, la dimensión ubicada en penúltimo lugar en cuanto a su contribución al modelo (.1621) fue la de marketing (MKT) influenciando con esta proporción, a la variable dependiente, lo anterior es probable por el estado incipiente en el que se encuentran el uso y la aplicación de las redes sociales en estos negocios de la región analizada.

\section{CONCLUSIONES}

Con base en lo expuesto anteriormente en este trabajo de investigación, se puede concluir que las ventajas que obtienen las mipymes al hacer uso de las redes sociales son identificar oportunidades para poder crear o mejorar productos o servicios, obtener nuevos clientes, recibir opiniones en tiempo real por parte de los consumidores, estar atentos a la competencia y a las tendencias, dar a conocer los productos o servicios a un mayor número de personas, es un marketing de bajo costo, entre otras.

El análisis multivariado permitió, a partir de un modelo de regresión lineal, medir los impactos de las dimensiones consideradas como razones o ventajas de unirse a las redes sociales y se evidenció que, tanto la dimensión de Comunicación e Innovación como la de Compartición de la Información, constituyen fuertes razones para que los empresarios consideren incorporar a sus negocios el uso de las redes sociales.

En la presente investigación se estableció la hipótesis de que las redes sociales representan para las mipymes una ventaja para adherirse a ellas. La cual se aceptó de manera contundente debido a los resultados que arrojó el modelo de regresión lineal donde la variable dependiente Ventajas de Adherirse a las Redes Sociales evidenció tener una relación lineal con las variables independientes IOP, SI, CI, RDC y MKT, las cuales manifestaron tener una influencia positiva en la misma.

Se evidenció que las mipymes perciben la importancia de la incorporación de sus negocios a las redes sociales al ser instrumento de impacto en la actualidad.

En la presente investigación se establecieron dos objetivos específicos, el primero de ellos fue determinar la intensidad de uso que dan las mipymes victorenses a las redes sociales. El primero fue alcanzado después de identificar que el $86 \%$ de las percepciones de los encuestados determinó 
una intensidad alta, por lo que permite concluir que es importante que las mipymes usen y apliquen estos medios digitales y así puedan obtener beneficios organizacionales. El segundo objetivo fue identificar cuáles fueron las dimensiones de mayor y menor impacto en las razones para adherirse de las mipymes victorenses a las redes sociales. Siendo la de Comunicación e Innovación (CI) la de mayor impacto; y la de menor impacto fue la de
Reducción de Costos (RDC).

El modelo de regresión se describe en la figura 1. De acuerdo con la evidencia lograda se refuerza la recomendación hacia las mipymes de que continúen haciendo esfuerzos para incorporar los beneficios de adherirse a las redes sociales y trabajar para reforzar las variables que aún no están bien desarrolladas

\section{REFERENCIAS}

Al-Badi , A., \& Al-Qayoudhi, W. (2014). Adoption of social networks in business: study of users and potential - users in Oman. International Business and Economics Research Journal, 13(2), 401-418.

Alvino, C. (2021). Estadísticas de la situación digital de México en el 2020-2021. Obtenido de: https://branch.com.co/marketing-digital/estadisticas-de-la-situacion-digital-de-mexico-en-el2020-2021/

Arceo-Moheno, G., Ramos-Méndez, E., Acosta-De la Cruz, J. (2019). Una visión de las competencias digitales de empresarios de Villahermosa, Tabasco. VinculaTégica. 1323-1335.

Arrubla, M. (2016). Finanzas y educación financiera en las empresas familiares Pymes. Sinapsis (8), 99- 118.

Ayyagari, M., Demirguc-Kunt, A. \& Maksimovic, V. (2017). SME Finance. 10.1596/1813-94508241.

Ballina, F., García-Perez-de-Lema, D., Martínez, F.J. (2016). La estrategia factor determinante del rendimiento de la MIPYME: Un estudio empírico en México, D.F. FAEDPYME International Review 4(7).

Banco Mundial. (2016). Los emprendedores y las pequeñas empresas impulsan el crecimiento económico $y$ crean empleos. Recuperado de: https://www.bancomundial.org/es/news/feature/2016/06/20/entrepreneurs-and-smallbusinesses-spur-economic-growth-and-create-jobs

Bocconcelli, R., Cioppi, M., Fortezza, F., Francioni, B., Pagano, A., Savelli, E., \& Splendiani, S. (2018). SMEs and Marketing: A Systematic Literature Review. International Journal of Management Reviews, 20(2), 227-254.

Borgerson, J. \& Miller, D. (2016). Scalable sociality and "How the world changed social media": conversation with Daniel Miller. Consumption Markets and Culture 19(6):1-14.

Bradley, A. (2017). Why Tech Is Our Best Bet to Spur U.S. Small Business Growth. Obtenido de https://blog.capterra.com/why-tech-is-our-best-bet-to-spur-u-s-small-business-growth/

CEPAL (2020). MIPYMES en América Latina. Naciones Unidas. Santiago de Chile. Recuperado de: 
https://repositorio.cepal.org/bitstream/handle/11362/44148/1/S1900361_es.pdf

Chiatchoua, C. \& Porcayo, A.Y. (2017) Las MIPyMEs en el mundo: elementos para una redefinición. Revista Debate Económico (17).

Dokyun, L., Kartik, Hosanagar \& Harikesh, S. Nair (2018). Advertising Content and Consumer Engagement on Social. Management Science, 1-27. doi: https://doi.org/10.1287/mnsc.2017.2902

Forbes (2018). Forbes. Obtenido de https://www.forbes.com.mx/pymesmexicanas-un-panoramapara-2018/

Franco, M., Haase, H., \& Pereira, A. (2016). Empirical study about the role of social networks in SME performance. Journal of Systems and Information Technology, 18(4), 383-403.

Goldberg, L. (2014). The Interactive Advertising Bureau. Obtenido de http://www.iab.net/about_the_iab/recent_press_releases/press_release_archive/pr ess_release/pr-041014

Granda, F., \& Hamann, F. (2015). Informality, Saving and Wealth Inequality in Colombia. United Sates: Interamerican Development Bank.

Kerlinger, F.N. \& Lee, H.B. (2002). Investigación del Comportamiento (4ª Ed.) Chile: McGraw Hill Interamericana.

Marc, C. (2015). Competencias Digitales, ESADE Universidad Ramón Llull. Recuperado de: http://mba.americaeconomia.com/blogs/esade/las-8-competencias-digitales-del-ceo/

Martínez, L. A. (2018). El Economista. Obtenido de https://www.eleconomista.com.mx/tecnologia/7-graficos-sobre-los-usuarios-deinterneten-Mexico-en-2018-20180517-0077.html

Medina, A. (2014). ¿Cuánto ha avanzado la publicidad online en México? Obtenido de http://www.altonivel.com.mx/42175-que-tanto-ha-avanzado-lapublicidad-online-enmexico.html

Muñoz, Á. E., \& Mayor, M. P. (2016). Las pyme en América Latina, Japón, la Unión Europea, Estados Unidos y los clúster en Colombia. Administración y Desarrollo, 45(1), 7-24.

Pelekais, E.A. \& Ortega D.V. (2020). Rendimiento empresarial sostenible para las micro, pequeñas y medianas empresas en Colombia. Revista Internacional de Cooperación y Desarrollo 2(7):104-118

Revilla, A.J., Perez-Luno, A., Nieto, M.J. (2016). Does family involvement in management reduce the risk of business failure? The moderating role of entrepreneurial orientation. Family Business Review, 29(4), 365-379.

Saavedra, M.L., Camarena, M.E. \& Tapia, B. (2017). Calidad para la competitividad en las micro, pequeñas y medianas empresas, de la Ciudad de México. Revista Venezolana de Gerencia 22(80), 551-575.

Sánchez Limón, M. L., \& De la Garza Cárdenas, M. H. (2018). Tecnologías de información y 
desempeño organizacional de las pymes del noreste de México. Revista Venezolana de Gerencia, 82, 298-313.

Santana, V. (2015). Uso de las redes sociales en apoyo al desarrollo de las Mypes mexicanas. Ciudad de México.

Torm, N. (2020). Gasto en seguridad social y rendimiento empresarial. El caso de las pymes en Indonesia. Revista Internacional del Trabajo 139(3).

Yang, M., Ren, Y., \& Adomavicius, G. (2019). Understanding User-Generated Content and Customer Engagement on Facebbok Business Pages. Information Systems Research, 1-17. 